\title{
Role of three key developmental variables in the emergence of long-range temporal correlations in a network of spiking neurons without plasticity
}

\author{
Nicholas Ward', Simon F Farmer ${ }^{2}$, Luc Berthouze ${ }^{1 *}$ \\ From Nineteenth Annual Computational Neuroscience Meeting: CNS*2010 \\ San Antonio, TX, USA. 24-30 July 2010
}

Neuronal avalanches in cortical slice cultures display statistical properties expected from a network operating at "criticality", i.e., an optimal state for input processing [1]. It has been suggested that balanced excitation and inhibition establishes criticality, and that this state is homeostatically regulated during development [2]. Here, we systematically characterized the behavior of a network of spiking neurons without plasticity when exploring three key variables of development: ratio of excitatory to inhibitory synaptic connections, range of excitatory conduction delays, number of synaptic connections.

We simulated a network of 1000 randomly connected neurons, with a variable ratio of excitatory neurons to inhibitory neurons $\left(N_{E} / N_{I}\right)$. There were $k$ outgoing synaptic connections per neuron, and no self- connections. The synaptic weights were fixed throughout. Inhibitory neurons only connected to excitatory neurons. Conduction delays were drawn from a uniform distribution $\left[1, d_{\text {max }}\right] \mathrm{ms}$ for excitatory neurons, fixed for inhibitory neurons $(1 \mathrm{~ms})$. Each neuron was governed by Izhikevich's approximation of a Hodgkin-Huxley-type neuronal model [3] such that excitatory neurons approximated cortical pyramidal neurons exhibiting regular spiking firing patterns and inhibitory neurons corresponded to cortical interneurons exhibiting fast spiking firing patterns. The network was jump-started at $\mathrm{t}=0$ by a single large injection of current.

The activity of the network was characterized in terms of (i) the presence of sustained activity, (ii) the presence of LRTCs as assessed by detrended fluctuation analysis [4] of the average activity of the network. Figure 1
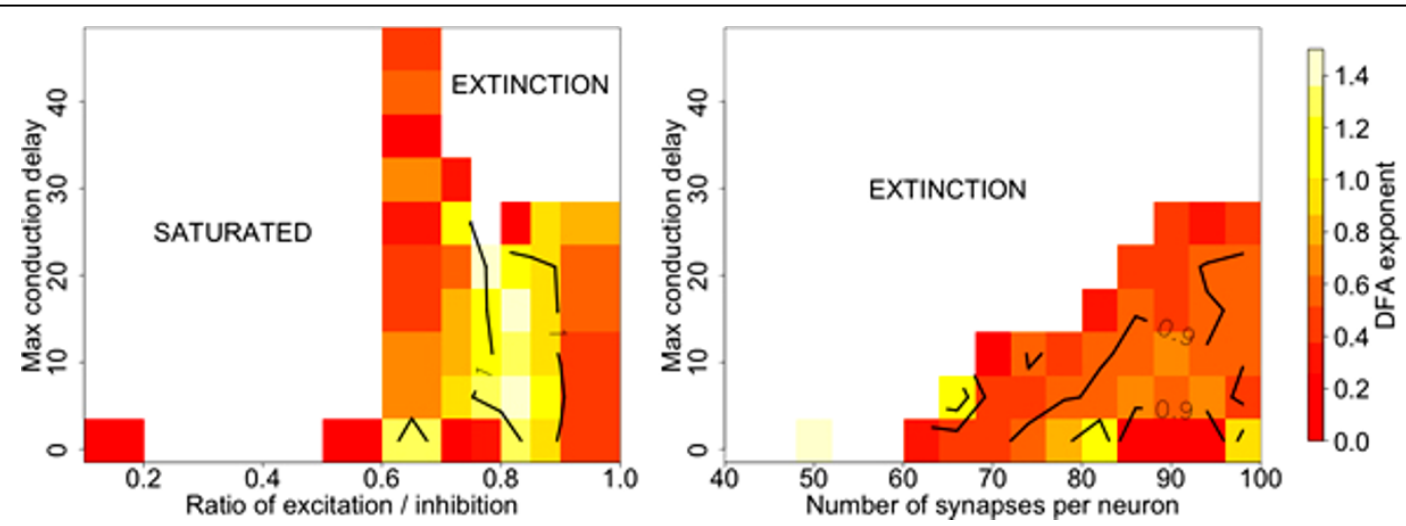

Figure 1 Regimes as a function of excitation/inhibition ratio, range of conduction delays and number of synapses.

* Correspondence: L.Berthouze@sussex.ac.uk

${ }^{1}$ Centre for Computational Neuroscience and Robotics, University of Sussex, Falmer, BN1 9QH, UK 
shows the different regimes obtained when systematically exploring the parameter space.

Consistently with predictions, non-trivial scaling exponents were only observed in a narrow range of ratio of excitation to inhibition. Deviations from this range either led to extinction of the activity or a regime largely dominated by fast-spiking oscillations (saturated regime). These results can be interpreted within the framework of branching processes, with LRTCs occurring when the branching parameter $\sigma \sim 1$, extinction when $\sigma<1$ and indefinite propagation of activity in the form of saturated oscillations when $\sigma>1$.

\section{Author details}

${ }^{1}$ Centre for Computational Neuroscience and Robotics, University of Sussex Falmer, BN1 9QH, UK. ${ }^{2}$ Institute of Neurology, University College London, London, WC1N 3BG, UK.

Published: 20 July 2010

\section{References}

1. Shew W, Yang H, Petermann T, Roy R, Plenz D: Neuronal avalanches imply maximum dynamic range in cortical networks at criticality. J Neurosci 2009, 29(49):15595-600.

2. Stewart C, Plenz D: Homeostasis of neuronal avalanches during postnatal cortex development in vitro. J Neurosci Methods 2008, 169(2):405-416.

3. Izhikevich E: Simple model of spiking neurons. IEEE Trans Neural Netw 2003, 14(6):1569-1572.

4. Peng C, Havlin S, Stanley H, Goldberger A: Quantification of scaling exponents and crossover phenomena in nonstationary heartbeat time series. Chaos 1995, 5:82-7.

doi:10.1186/1471-2202-11-S1-P29

Cite this article as: Ward et al:: Role of three key developmental variables in the emergence of long-range temporal correlations in a network of spiking neurons without plasticity. BMC Neuroscience 2010 11(Suppl 1):P29.

\section{Submit your next manuscript to BioMed Central} and take full advantage of:

- Convenient online submission

- Thorough peer review

- No space constraints or color figure charges

- Immediate publication on acceptance

- Inclusion in PubMed, CAS, Scopus and Google Scholar

- Research which is freely available for redistribution

Submit your manuscript at www.biomedcentral.com/submit 